\title{
Development and benchmarking of codes for simulation of beam-beam effects at the LHC
}

\author{
F. Schmidt, CERN \\ A. Valishev, FNAL \\ Y. Luo, BNL
}

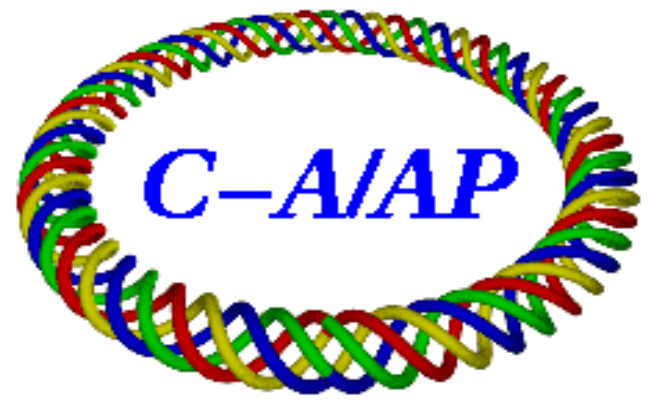

\section{Collider-Accelerator Department Brookhaven National Laboratory \\ Upton, NY 11973}

Notice: This document has been authorized by employees of Brookhaven Science Associates, LLC under Contract No. DE-AC02-98CH10886 with the U.S. Department of Energy. The United States Government retains a nonexclusive, paid-up, irrevocable, world-wide license to publish or reproduce the published form of this document, or allow others to do so, for United States Government purposes. 


\title{
$\mathrm{BNL} \mathrm{C}-\mathrm{A} / \mathrm{AP} / 443$
}

\section{DEVELOPMENT AND BENCHMARKING OF CODES FOR SIMULATION OF BEAM-BEAM EFFECTS AT THE LHC*}

\author{
F. Schmidt, CERN, Geneva, Switzerland \\ A. Valishev, FNAL, Batavia, IL 60510, USA \\ Y. Luo, BNL, Upton, NY 11973-5000, USA
}

January 11, 2012

\begin{abstract}
Beam dynamics in modern hadron colliders is strongly affected by effects of beam-beam interactions, which are complex phenomena and generally require extensive numerical simulations for accurate evaluation. In this note we describe the development of codes used for simulations of beam-beam effects at the LHC. We benchmark the codes on several test cases and report the results of this comparison.
\end{abstract}

\section{DESCRIPTION OF CODES}

\section{$1.1 \quad$ Lifetrac}

The code is described in more detail in the Proceedings of PAC05 [1]. Below a brief summary of the code features is given. Lifetrac is a weak-strong beam-beam code that was originally created for simulating equilibrium distributions in electron-positron colliders (circa 1995). Eventually, the author added the functionality to simulate non-equilibrium distributions (2003-2004), making it a conventional macro particle tracking code. Lifetrac tracks a bunch of particles through the machine lattice and beam-beam interactions. The major design principles are the following

- Machine lattice is comprised of 6D linear maps and thin multipoles.

- Linear maps are specified with the use of beta-functions (conventional or coupled), phase advances and $M_{56}$. A Perl script will take MAD-X TWISS output and convert it into a form readable by Lifetrac.

- Thin multipoles are read from MAD-X with a Perl script

- RF cavity (one or several per turn) is a sinusoidal kick.

*Work supported by the US DOE through the US LHC Accelerator Research Program (LARP) 
- Because the machine arcs are linear maps without chromatic dependence, there are two special methods for treatment of the lattice chromaticity. The linear (tune) chromaticity is implemented via an additional phase advance dependent on the particle's momentum, applied one or several times per turn. The second order (beta-function) chromaticity is added in the form of "chromatic drifts". They are added before and after the important IPs. For more details refer [1]. Both methods are symplectic.

- Beam-beam elements are 6D symplectic kicks (Hirata's formulae), their locations and parameters (beta-functions of the strong bunch, separation of the colliding bunches, crossing angle) are also read from MAD-X output. The main IPs are sliced longitudinally into arbitrary number of slices (12 was determined to work well for the Tevatron case of $\sigma_{z} / \beta^{*} \simeq 1$ ). Long-range IPs are not sliced (thin).

- An important feature for the Tevatron was the introduction of various noises in the form of random kicks applied once per turn. These kicks are specified using a correlation matrix.

- We typically track $10^{\prime} 000$ particles for $10^{6}-10^{7}$ turns. Because the number of particles is not sufficient to describe beam emittances and lifetime with good precision, we enhance it by

- Averaging the density distribution over the simulation step, usually 10 '000 turns.

- Using weighted distribution with more particles in the tails.

The code reads in all particles and then sends them to parallel nodes for tracking over the step. This is done via MPI. During a step nodes don't talk to each other. Then, at the end of each step the particle coordinates are collected by the head process to perform averaging and also for saving the simulation snapshot.

\section{$1.2 \quad$ Sixtrack}

The well-known SixTrack code [2] treats motion in the full 6d phase space [3] with the extended Hamiltonian, i.e. it should be used for large proton accelerators where the relative momentum deviation deltap is not excessively large. The 4D BB force is treated by the usual formalism 44 while the $6 \mathrm{D}$ case is treated à la Hirata [5] and updated by Ripken to include arbitrary crossing planes and coupling [6]. The code has been optimized for speed and an elaborate run environment has been set-up to allow for massive tracking studies $[7$.

\subsection{SimTrack}

Sim Track is a simple $\mathrm{C}++$ library designed for numeric particle tracking in high energy accelerators. It adopts the $4^{\text {th }}$ order symplectic integrator 8,9 for the optical transport in magnetic elements. The 4-D and 6-D weak-strong beam-beam treatments [4, 5] are integrated in it for the beam-beam studies. The optical transfer through magnetic elements in SimTrack was bench-marked with TracyII and its 4D and 6D weak-strong beam-beam calculations were benchmarked with BBSIMC [10].

SimTrack provides versatile functions to manage elements and lines. It supports a large range of types of elements. New types of element can be easily created in the library. SimTrack calculates TWISS, coupling and fits tunes, chromaticities and corrects closed orbits. AC dipole, AC multipole, and electron lens are all available in this library. SimTrack allows access and change of element parameters during tracking. 
SimTrack has been extensively used for the nonlinear beam dynamics studies for the RHIC Au ion and polarized proton operation and for the RHIC head-on beam-beam compensation. Functions to calculate tune footprint, tune and amplitude diffusion, and dynamic aperture are provided. It also calculates non-linear resonance driving terms and detuning from sextupoles and octupoles using analytic formulae.

To study the proton beam lifetime with head-on beam-beam compensation scheme in RHIC, Sim Track is used to calculate the proton particle loss and emittance growth with about 5'000 macroparticles up to $2 \times 10^{6}$ turns. To speed up the particle tracking, the integration steps of dipoles and quadrupoles are reduced. The tunes and chromaticities need to be re-matched before tracking.

To save the computing time involved in the lifetime calculation, we adopt a hollow Gaussian distribution for the initial proton particle coordinates. The advantage of this method is that there are more macro-particles in the bunch tail. With this method the particle loss rate below $1 \% /$ hour or $0.007 \%$ particle loss in $2 \times 10^{6}$ turns can be detected with the RHIC lattices.

For the emittance determination, same as Lifetrac, we calculate every 10'000 turns, $\left\langle x^{2}\right\rangle$, $\left\langle y^{2}\right\rangle$ and $\left\langle z^{2}\right\rangle$ with the coordinates of all macro-particles. In doing so, an emittance change of $0.03 \%$ can be detected with $2 \times 10^{6}$ turns. 


\section{BENCHMARKING WITHOUT BEAM-BEAM}

Since the model of the LHC optics is quite complex, it is essential to establish the code agreement in single particle tracking without beam-beam interactions. In this section we present the results of comparison of SixTrack and Lifetrac in the 4D and $6 \mathrm{D}$ phase space. The criteria for comparison are the distance in phase space between two initially close particles, the survival plots and the dynamic aperture as a function of $\mathrm{X}-\mathrm{Y}$ phase space angle. It can be seen from Figures 1 14 that agreement between the two codes is not perfect but the difference in reported dynamical aperture does not exceed $20 \%$. The model of nonlinearities in Lifetrac includes only final focus triplet magnetic errors and lattice sextupoles and thus generally gives larger dynamical aperture values.

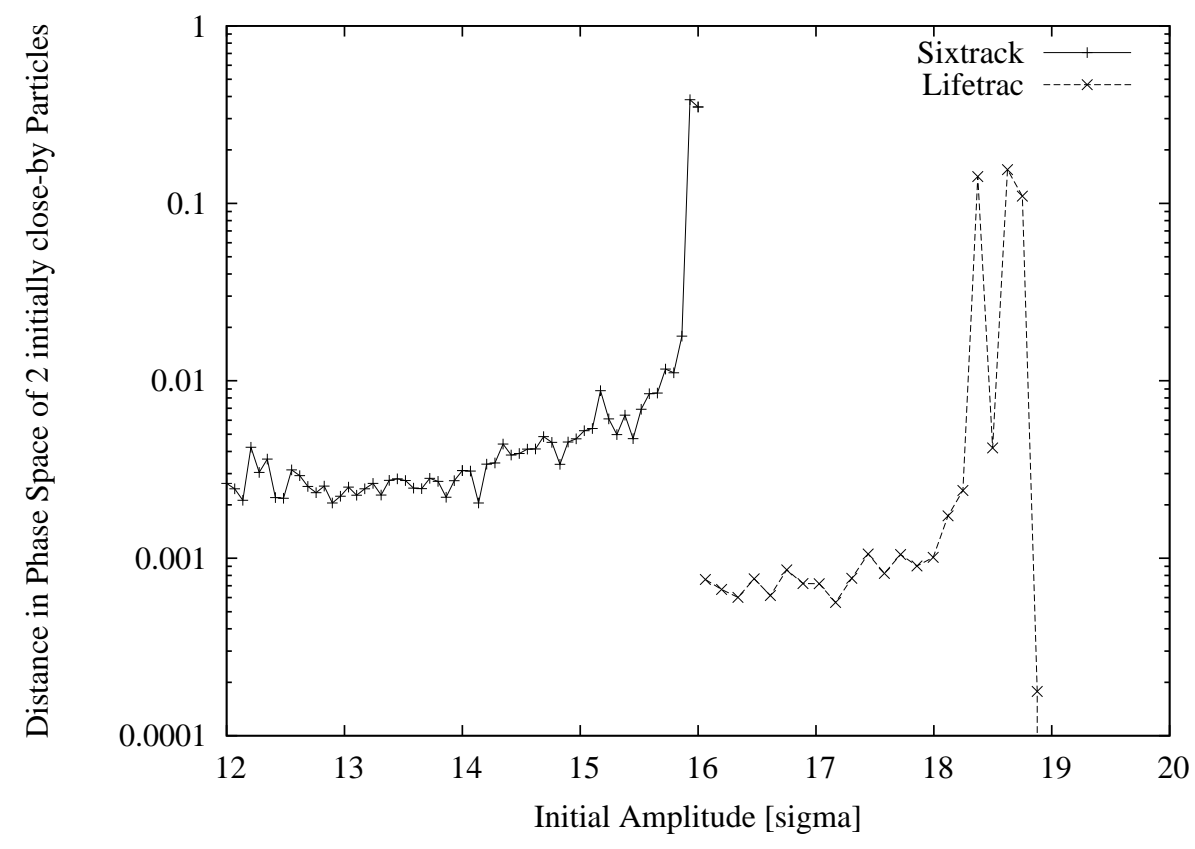

Figure 1: Distance in phase space of two initially close particles after $10^{6}$ turns of tracking vs. the initial amplitude. Beam-beam interaction is off, particles' momentum deviation is 0 (4D case). Initial angle in $\mathrm{Ax}$, Ay plane is 15 degrees. 


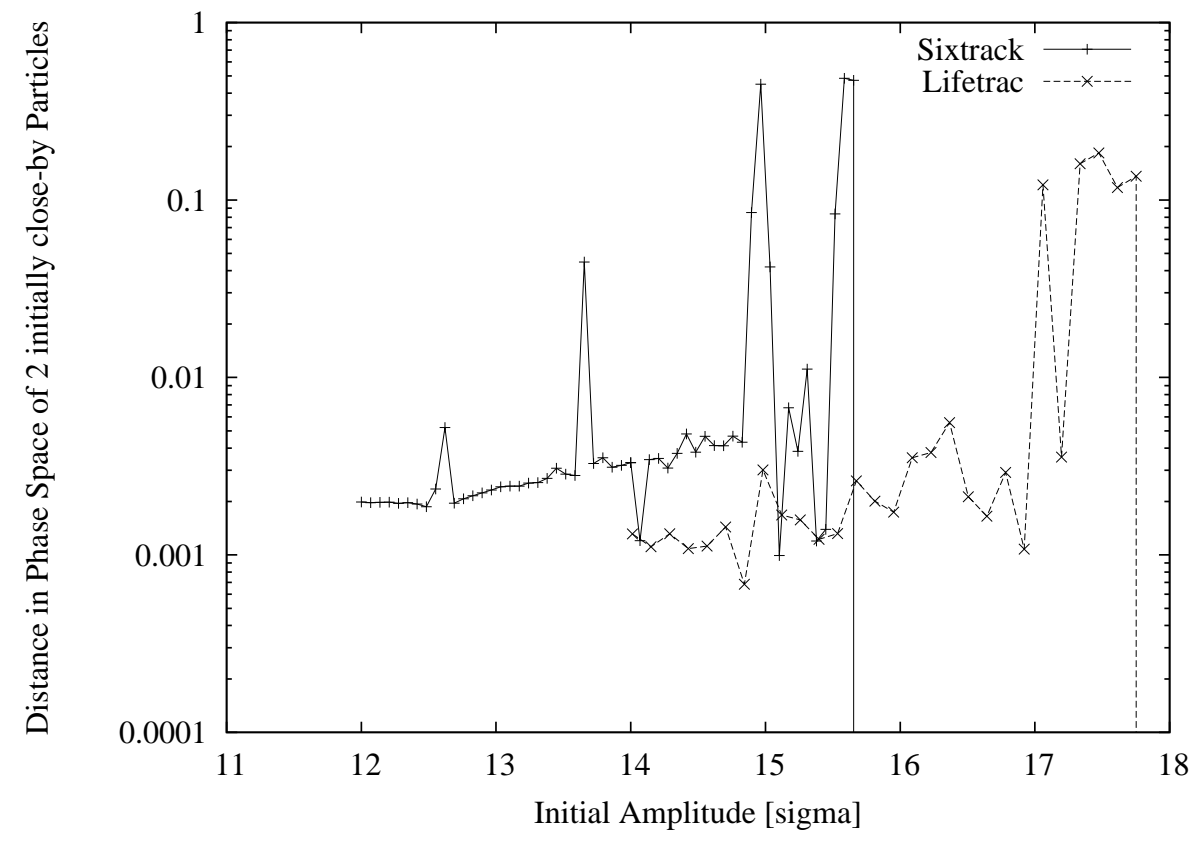

Figure 2: Distance in phase space of two initially close particles after $10^{6}$ turns of tracking vs. the initial amplitude. Beam-beam interaction is off, particles' momentum deviation is 0 ( $4 \mathrm{D}$ case). Initial angle in Ax, Ay plane is 45 degrees. 


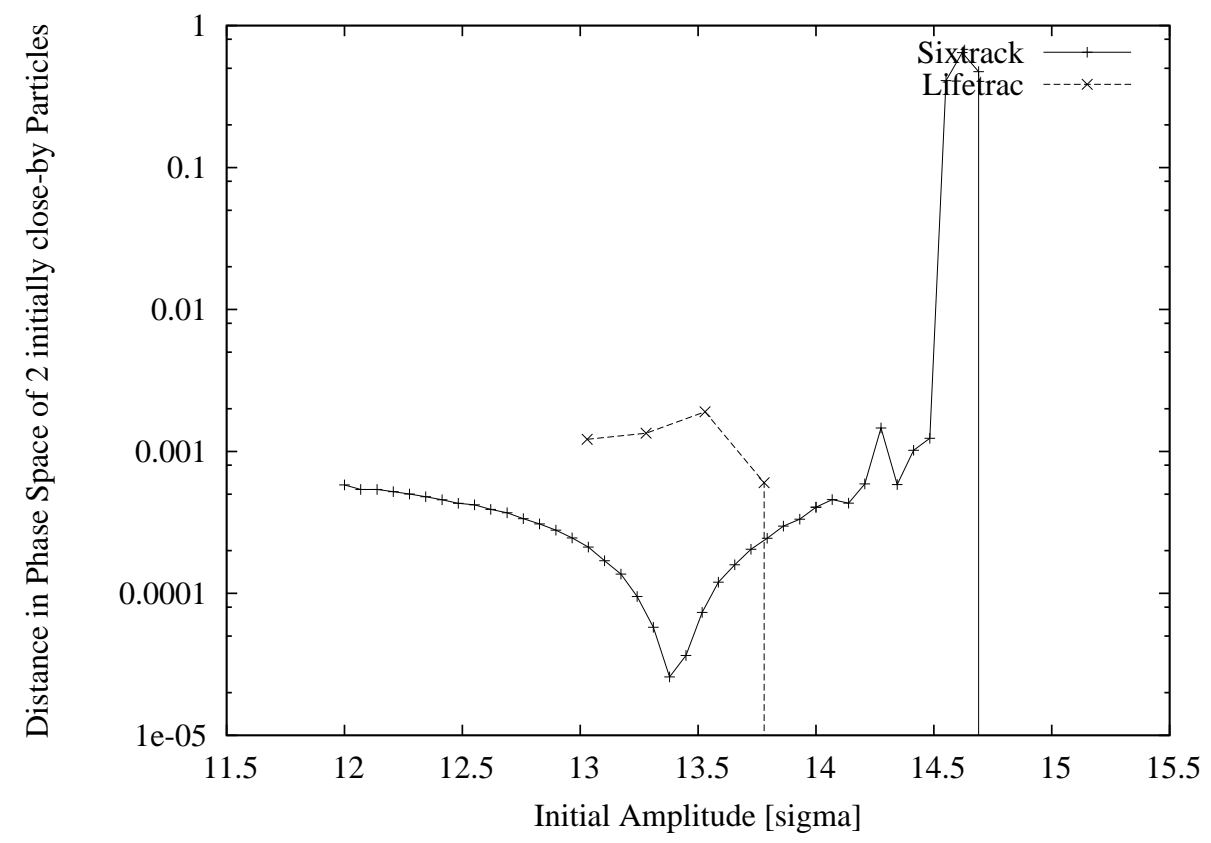

Figure 3: Distance in phase space of two initially close particles after $10^{6}$ turns of tracking vs. the initial amplitude. Beam-beam interaction is off, particles' momentum deviation is 0 ( $4 \mathrm{D}$ case). Initial angle in Ax, Ay plane is 75 degrees. 


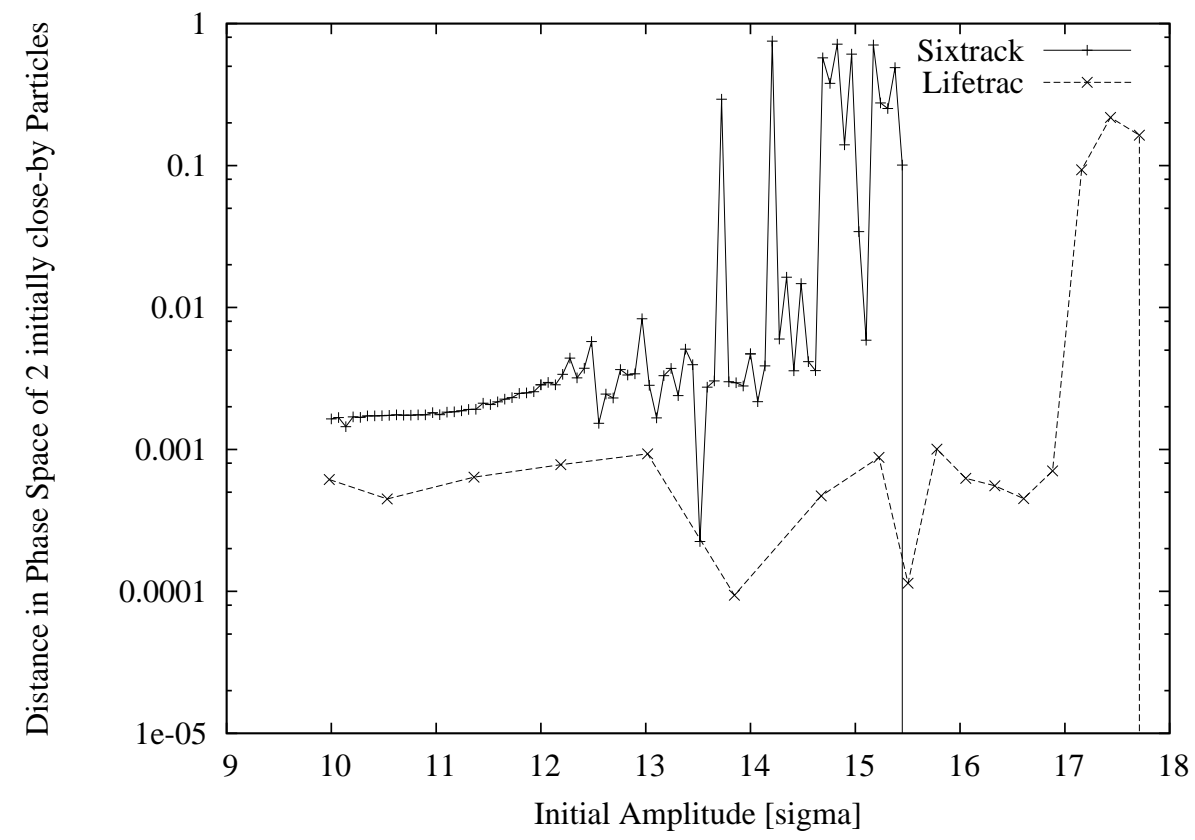

Figure 4: Distance in phase space of two initially close particles after $10^{6}$ turns of tracking vs. the initial amplitude. Beam-beam interaction is off, particles' momentum deviation is 0.00027 (6D case). Initial angle in Ax, Ay plane is 15 degrees. 


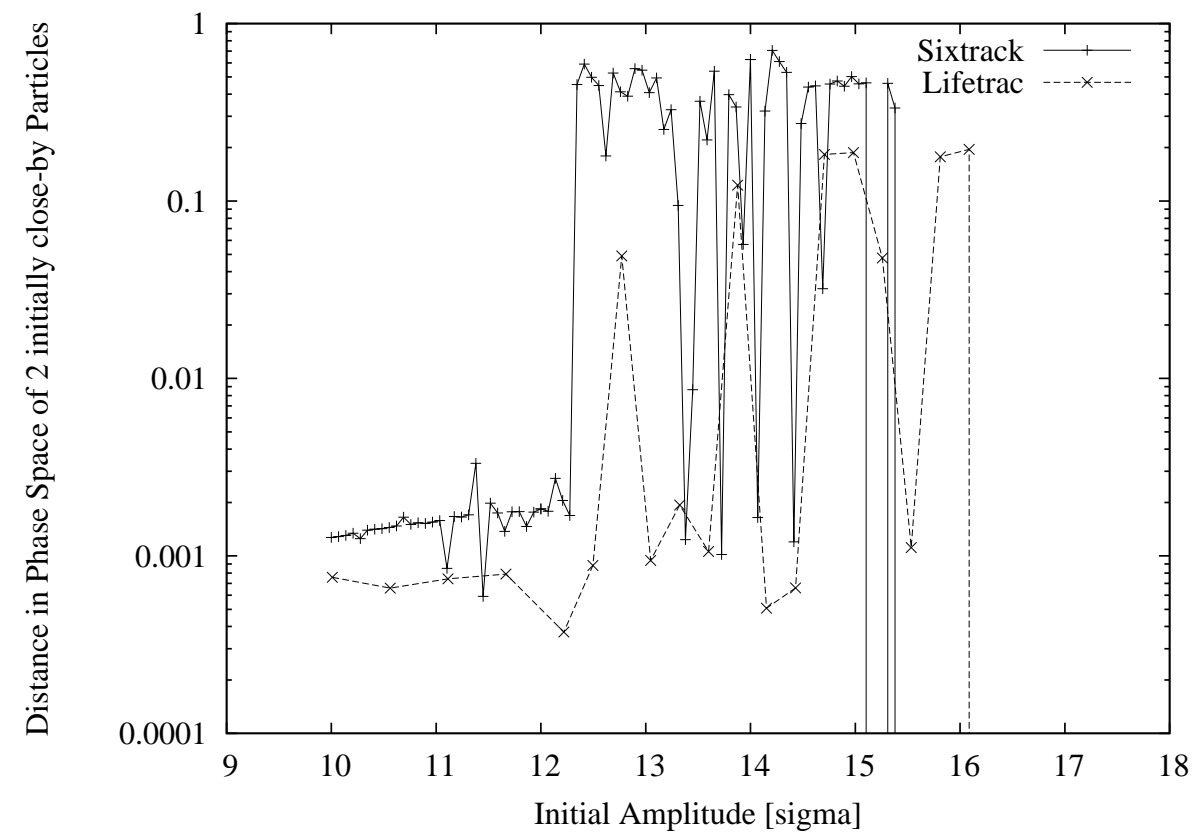

Figure 5: Distance in phase space of two initially close particles after $10^{6}$ turns of tracking vs. the initial amplitude. Beam-beam interaction is off, particles' momentum deviation is 0.00027 (6D case). Initial angle in Ax, Ay plane is 45 degrees. 


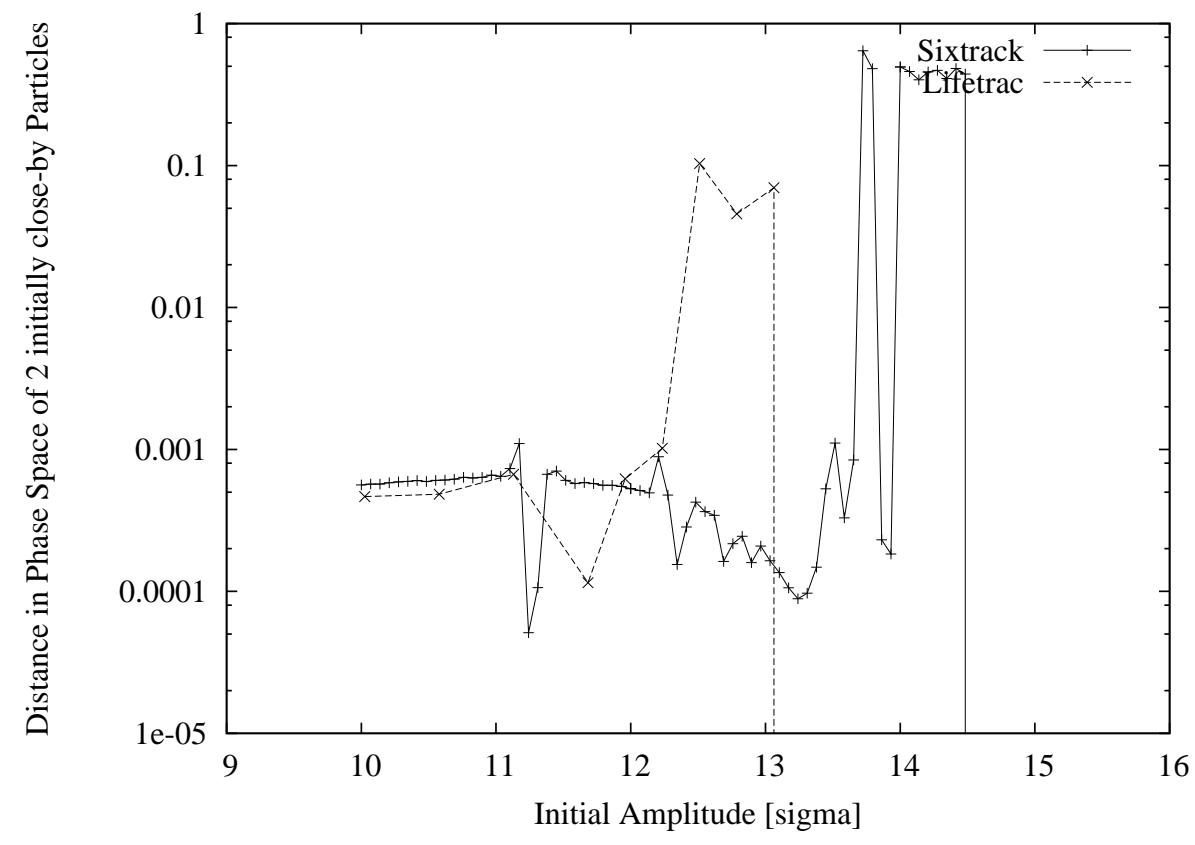

Figure 6: Distance in phase space of two initially close particles after $10^{6}$ turns of tracking vs. the initial amplitude. Beam-beam interaction is off, particles' momentum deviation is 0.00027 (6D case). Initial angle in $\mathrm{Ax}$, Ay plane is 75 degrees. 


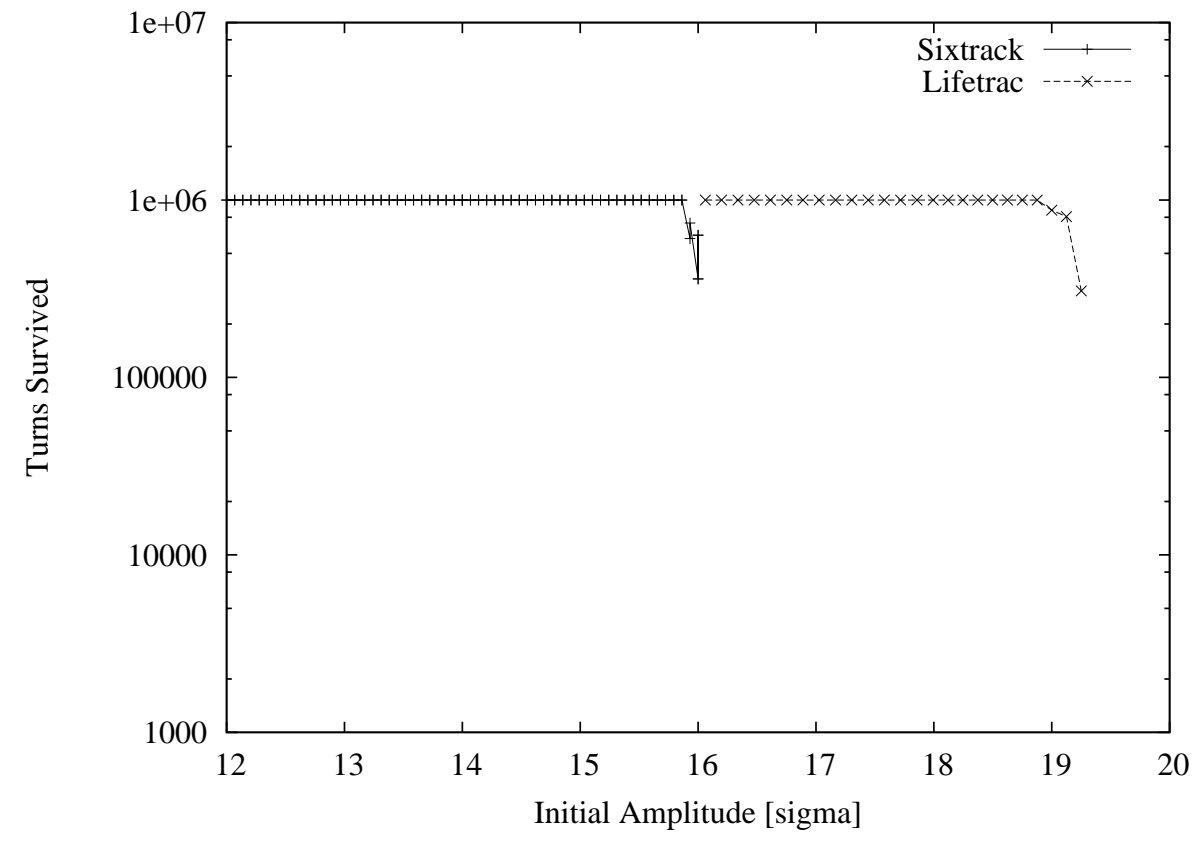

Figure 7: Particle survival time in turns vs. the initial amplitude. Beam-beam interaction is off, particles' momentum deviation is 0 ( $4 \mathrm{D}$ case). Initial angle in $\mathrm{Ax}$, Ay plane is 15 degrees. 


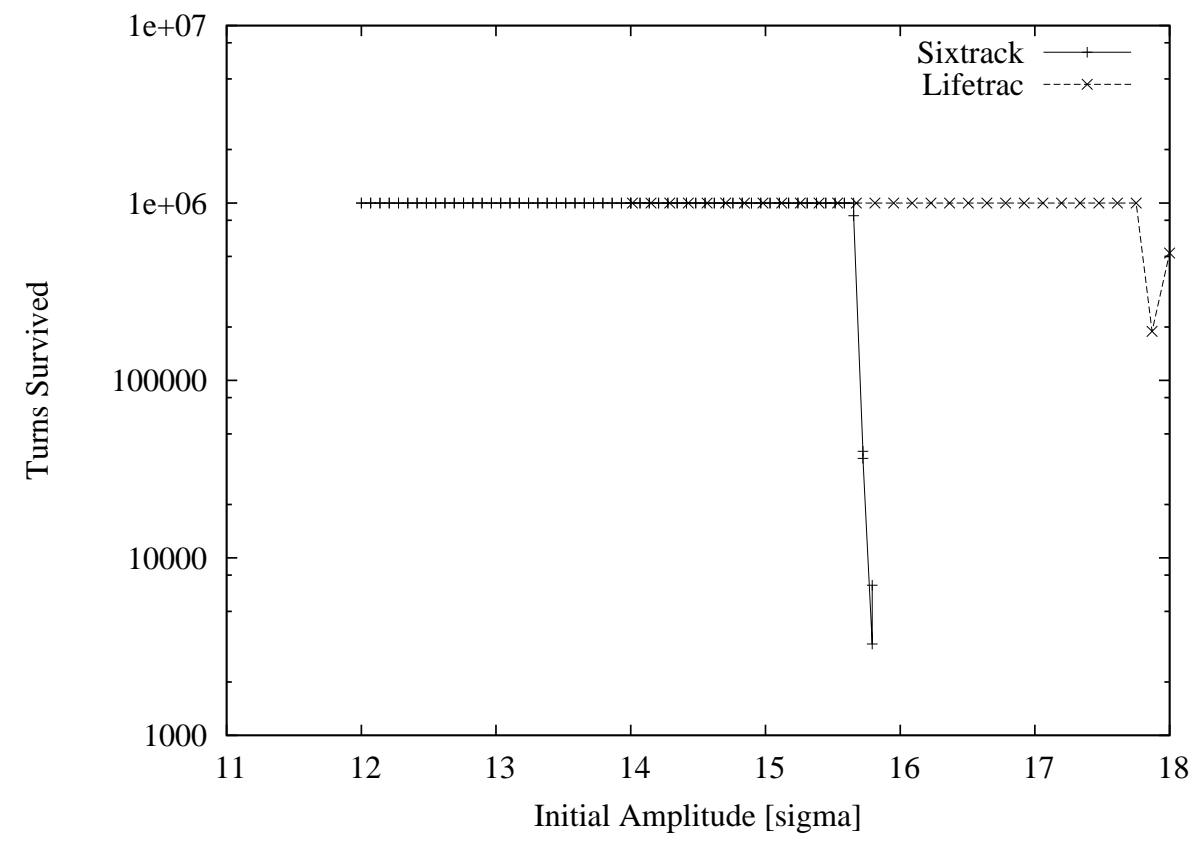

Figure 8: Particle survival time in turns vs. the initial amplitude. Beam-beam interaction is off, particles' momentum deviation is 0 ( $4 \mathrm{D}$ case). Initial angle in $\mathrm{Ax}$, Ay plane is 45 degrees. 


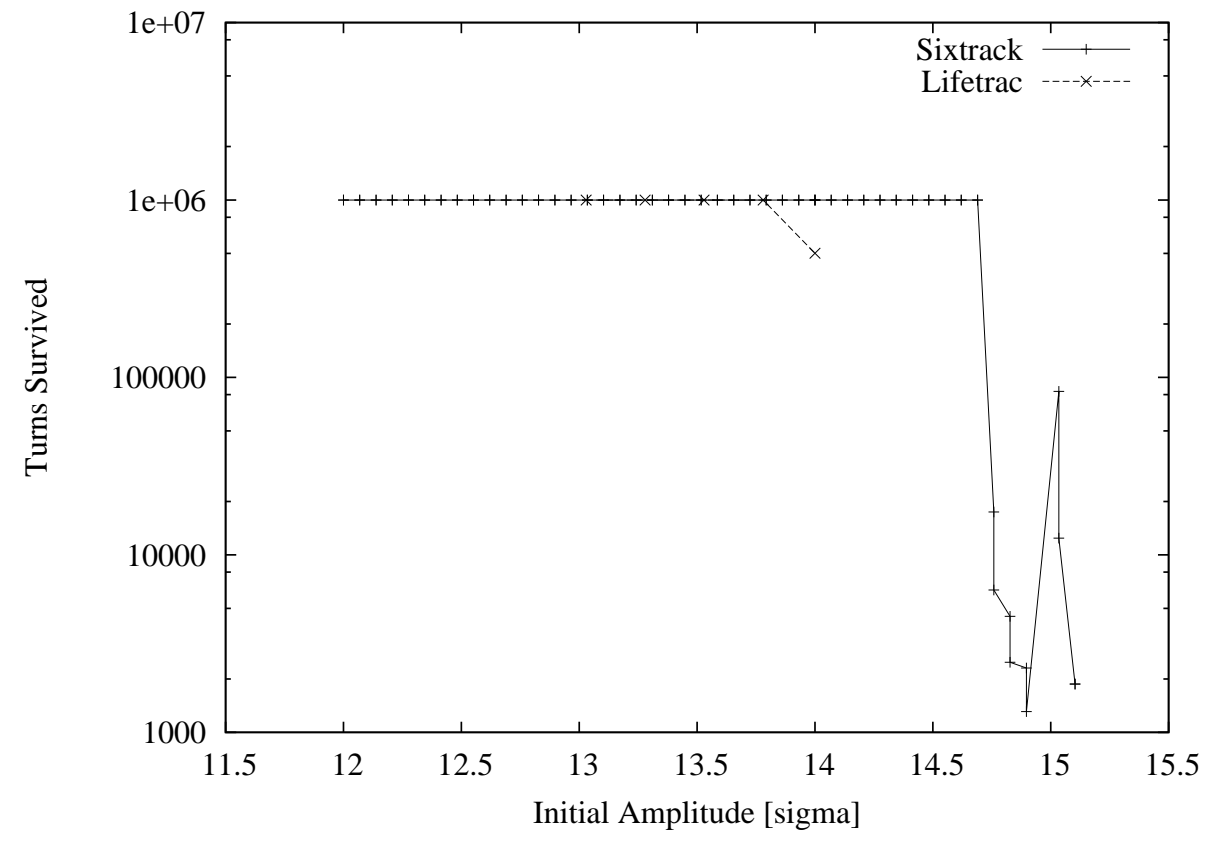

Figure 9: Particle survival time in turns vs. the initial amplitude. Beam-beam interaction is off, particles' momentum deviation is 0 ( $4 \mathrm{D}$ case). Initial angle in $\mathrm{Ax}$, Ay plane is 75 degrees. 


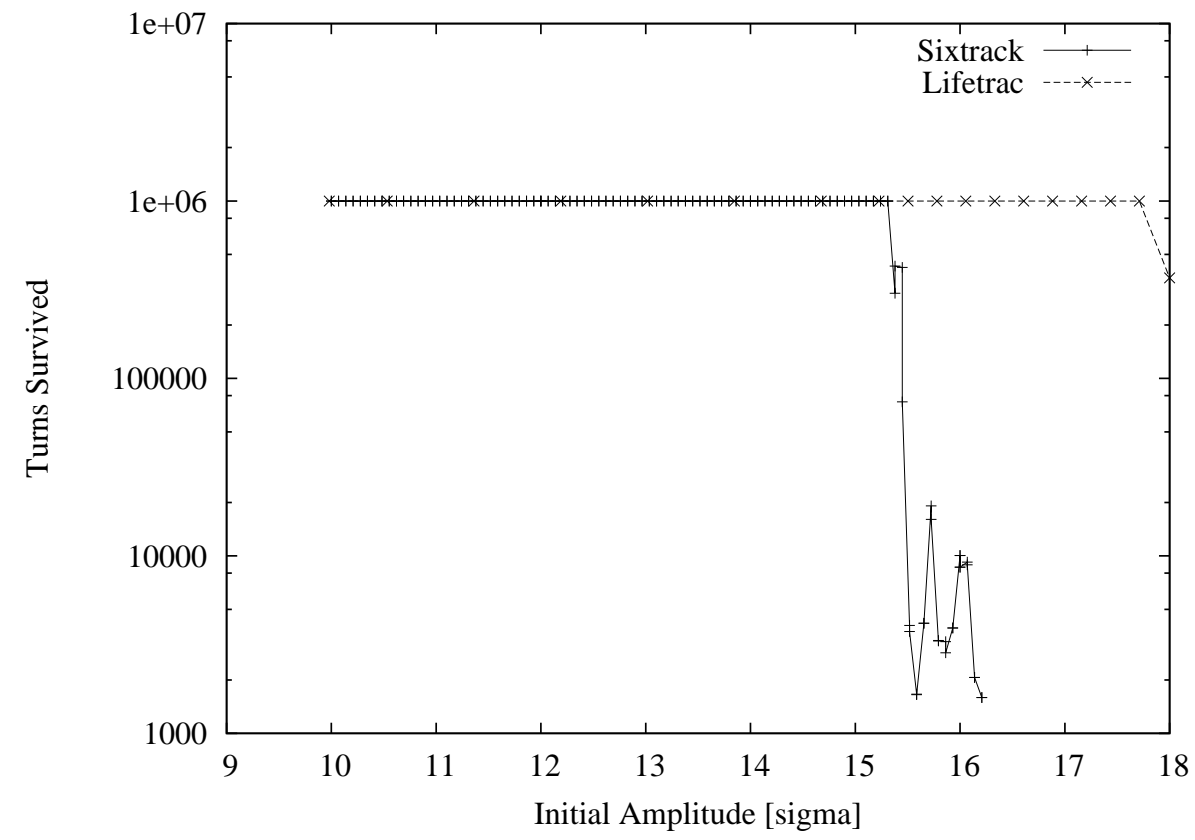

Figure 10: Particle survival time in turns vs. the initial amplitude. Beam-beam interaction is off, particles' momentum deviation is 0.00027 (6D case). Initial angle in Ax, Ay plane is 15 degrees. 


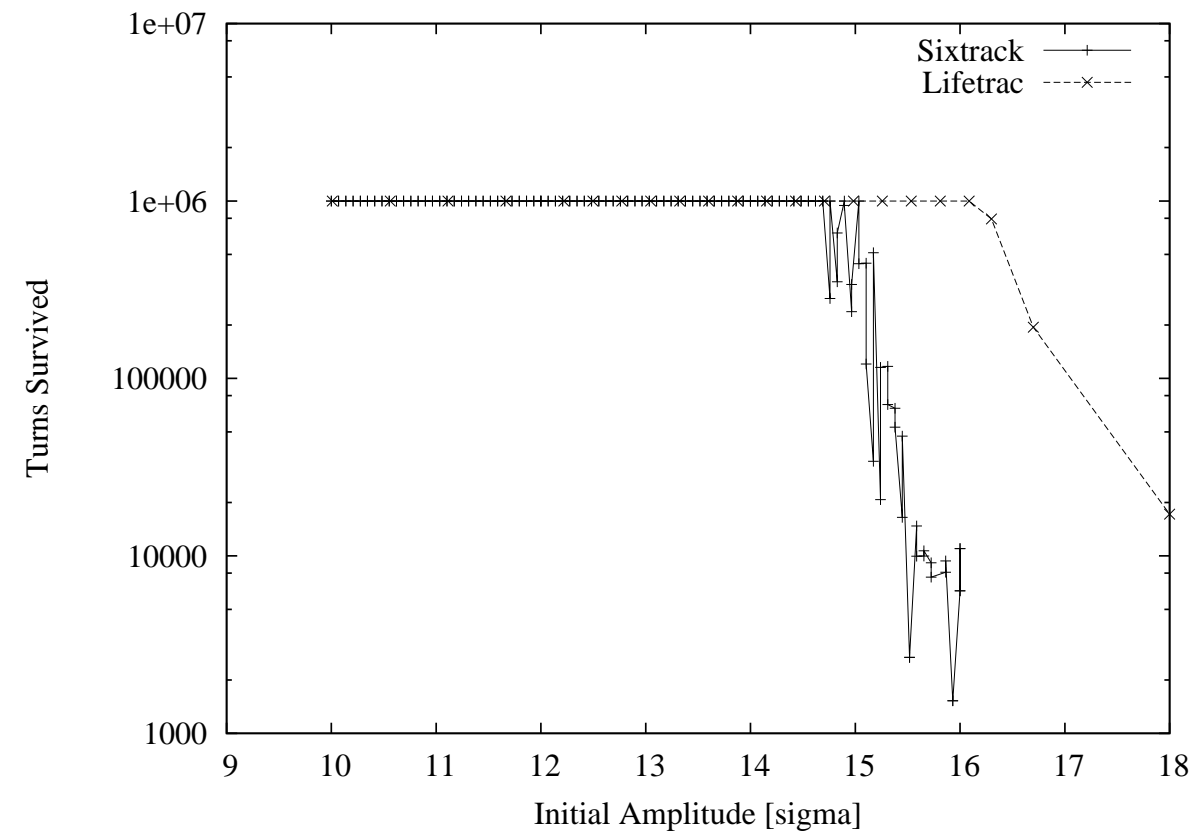

Figure 11: Particle survival time in turns vs. the initial amplitude. Beam-beam interaction is off, particles' momentum deviation is 0.00027 (6D case). Initial angle in Ax, Ay plane is 45 degrees. 


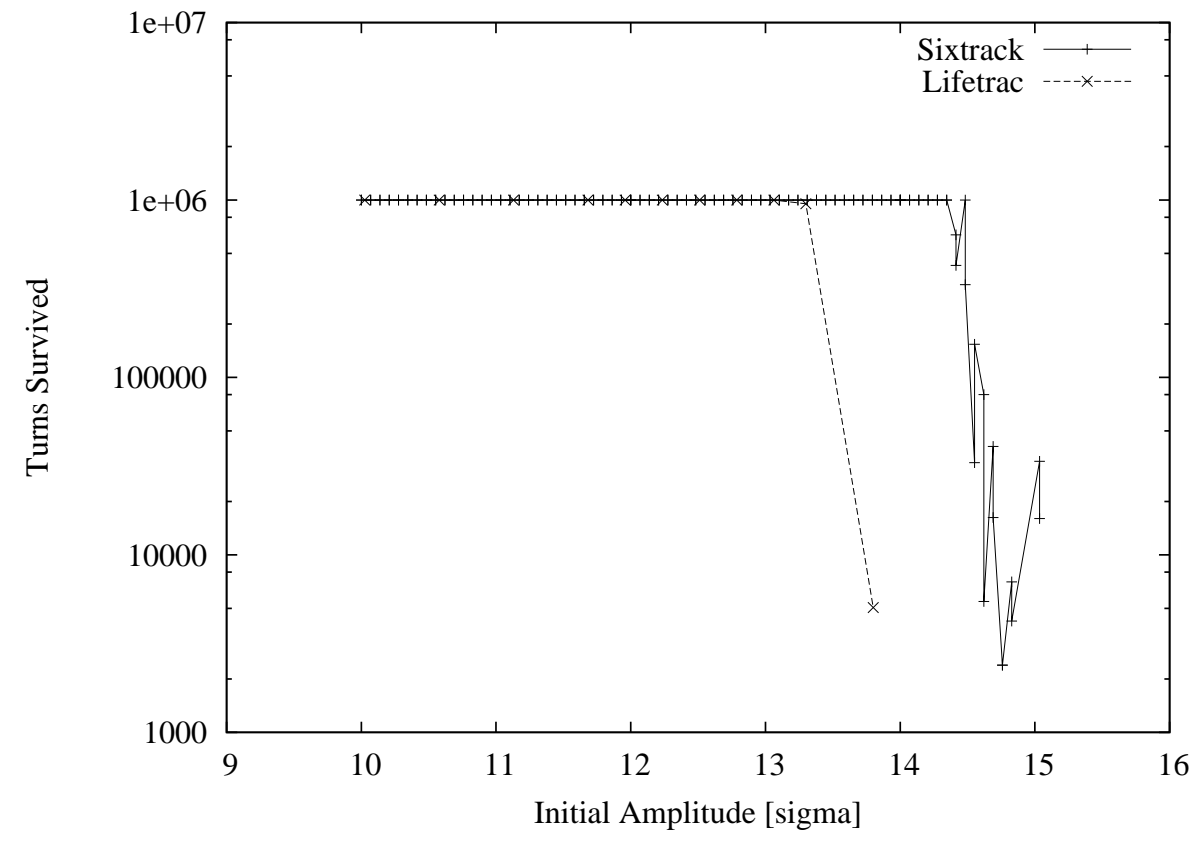

Figure 12: Particle survival time in turns vs. the initial amplitude. Beam-beam interaction is off, particles' momentum deviation is 0.00027 (6D case). Initial angle in Ax, Ay plane is 75 degrees. 


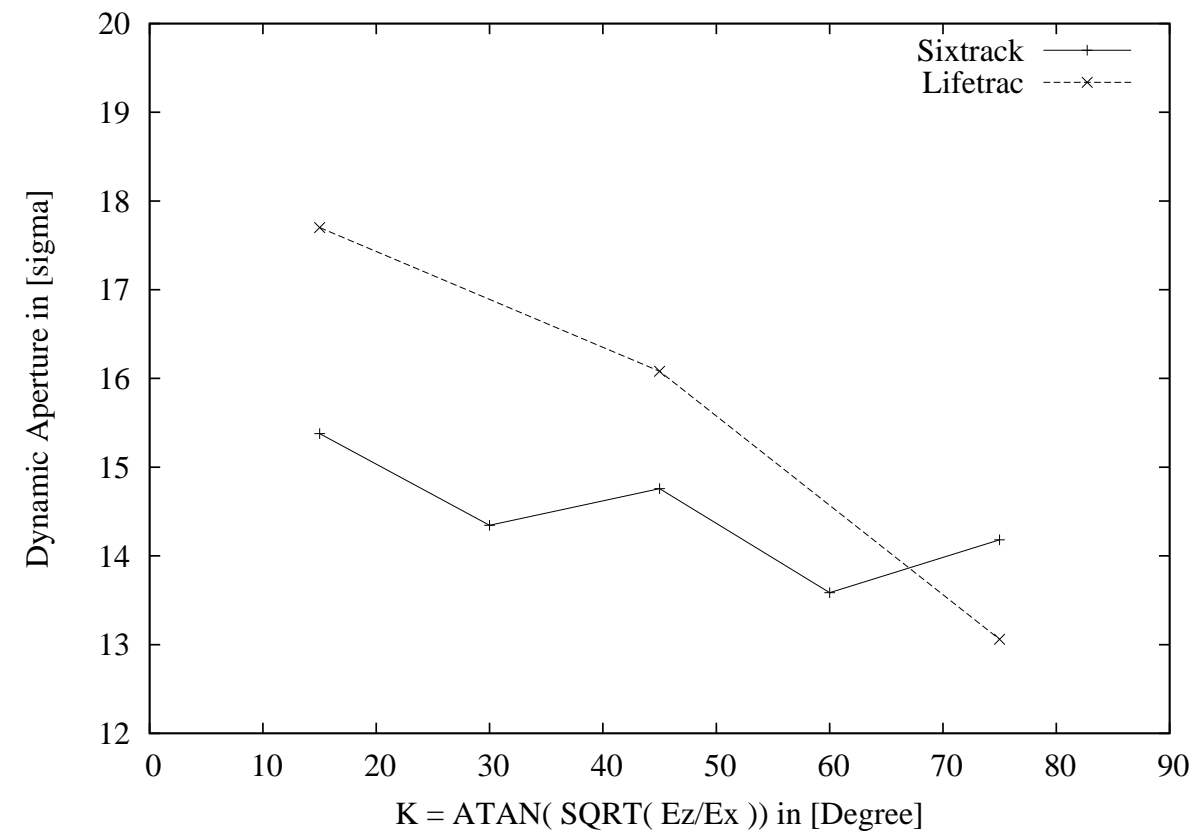

Figure 13: Dynamic aperture in beam sigma vs. initial angle in Ax, Ay plane. Beam-beam interaction is off, particles' momentum deviation is 0.00027 (6D case). 


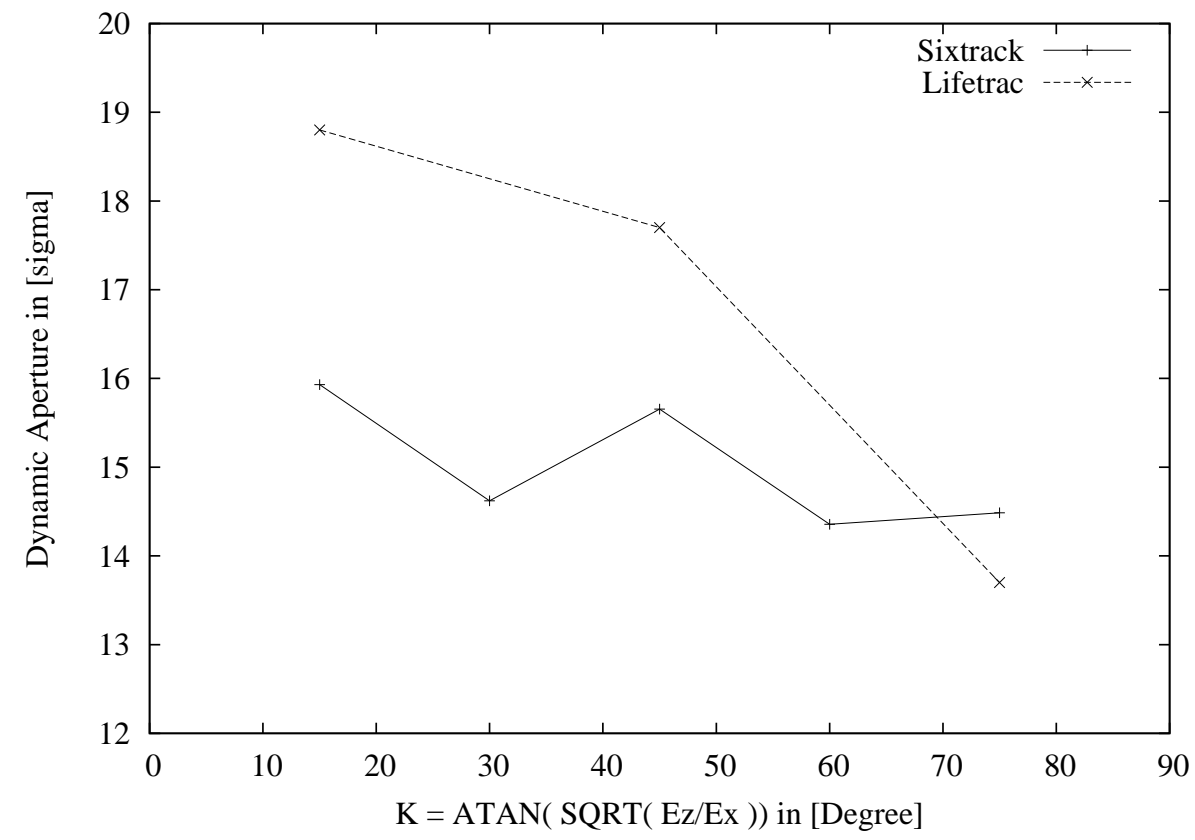

Figure 14: Dynamic aperture in beam sigma vs. initial angle in Ax, Ay plane. Beam-beam interaction is off, particles' momentum deviation is 0 ( $4 \mathrm{D}$ case). 


\section{BENCHMARKING WITH BEAM-BEAM}

In this sections we compare SixTrack and Lifetrac, in $4 \mathrm{D}$ and including $\mathrm{BB}$, with respect to the distance in phase space and the survival plot. Again, Lifetrac gives a larger value of dynamical aperture, which however is within $20 \%$ of the SixTrack prediction.

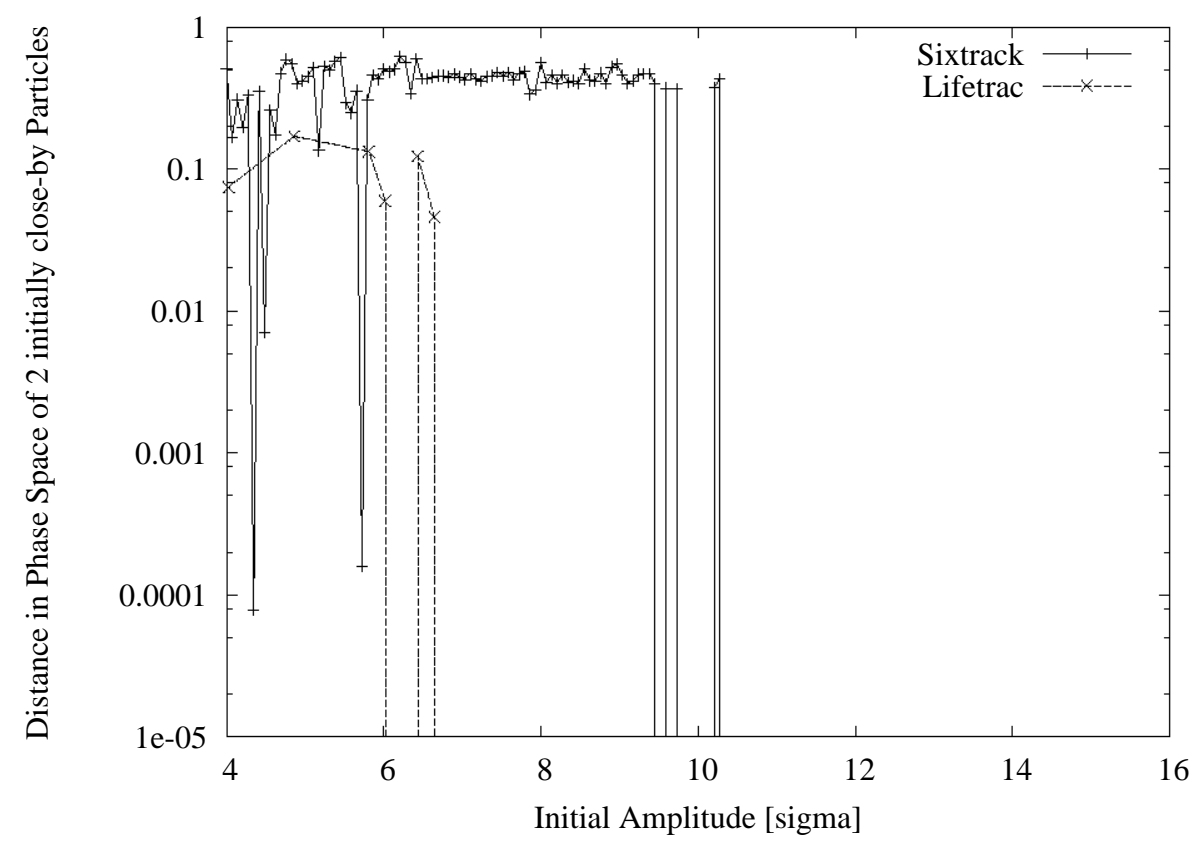

Figure 15: Distance in phase space of two initially close particles after $10^{6}$ turns of tracking vs. the initial amplitude. Beam-beam interaction is on, particles' momentum deviation is 0 ( $4 \mathrm{D}$ case). Initial angle in $\mathrm{Ax}$, Ay plane is 45 degrees. 


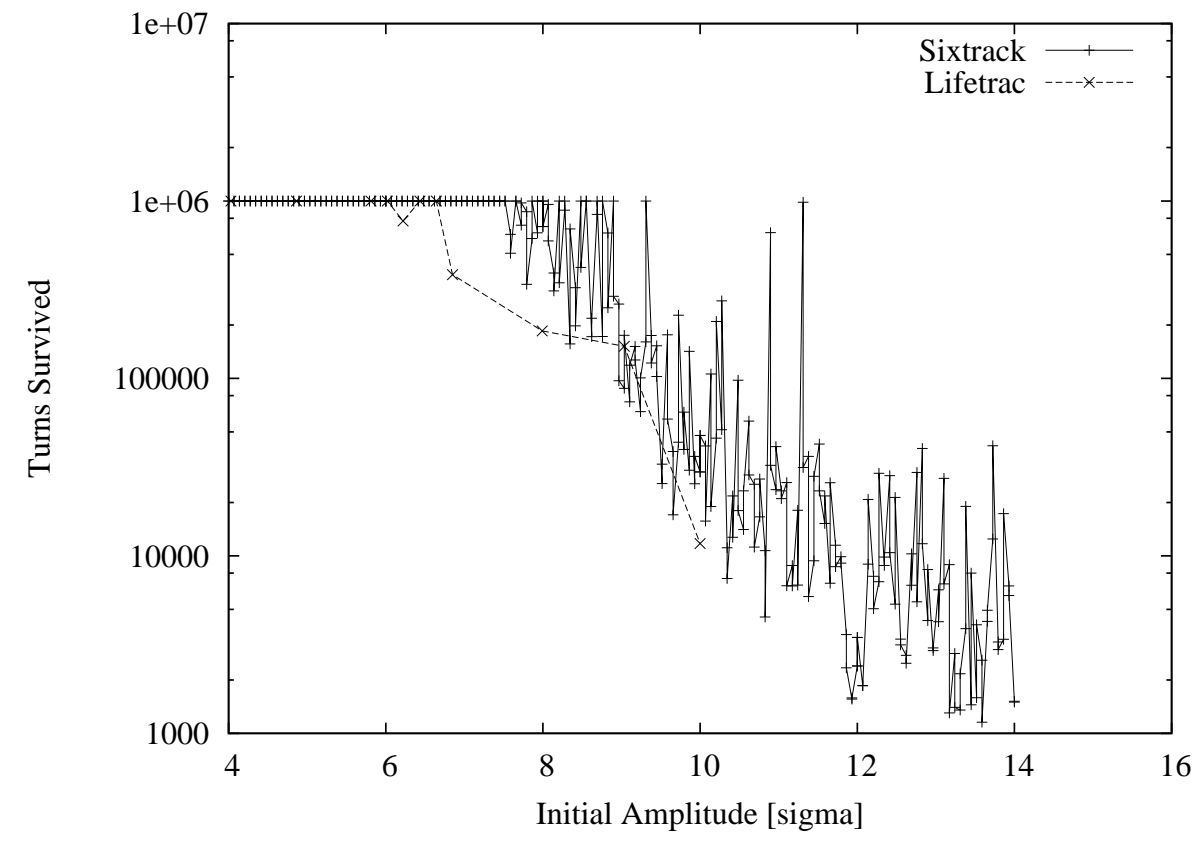

Figure 16: Particle survival time in turns vs. the initial amplitude. Beam-beam interaction is on, particles' momentum deviation is 0 ( $4 \mathrm{D}$ case). Initial angle in $\mathrm{Ax}$, Ay plane is 45 degrees. 


\section{LHC tune footprints}

The first three plots in this sections show the LHC head-on BB without and with the long range BB. The fourth plots shows the comparison with Lifetrac for the head-on BB. The captions describes the details.

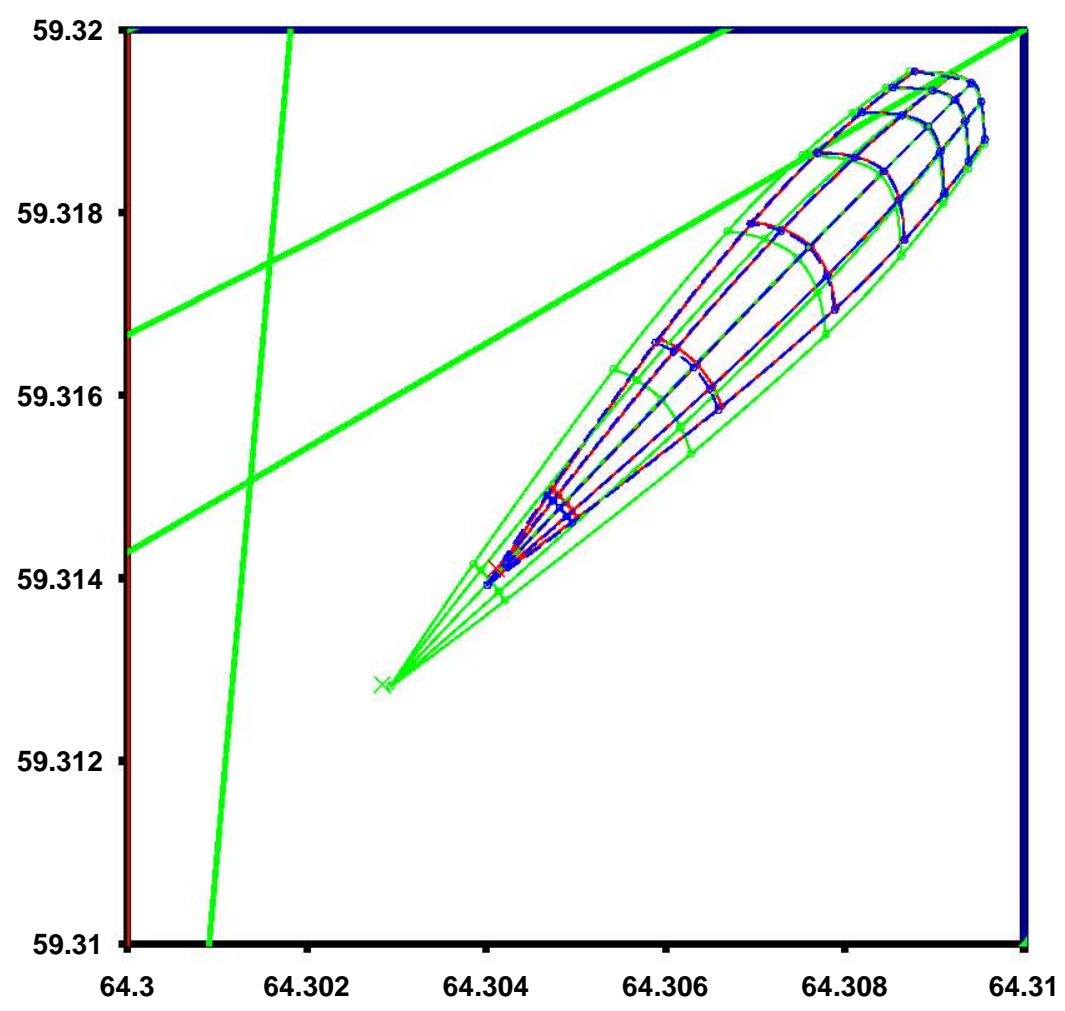

Figure 17: LHC head-on BB in IP1 \& IP5 with nominal crossing angle (deltap $=0$ ). Green depicts the $4 \mathrm{D}$ case of a single head-on BB per IP while in red the $6 \mathrm{D}$ case (13 slices) is shown. The large green and red "X" are the theoretical tuneshifts for the nominal and crossing angle case (see Eq2). They are in excellent agreement within the precision of the settings of the parameters. In blue is overlaid the $4 \mathrm{D}$ BB tune footprint where the head-on is manually distributed over 5 slices properly placed longitudinally. The close agreement with the $6 \mathrm{~d}$ case justifies why for the LHC 4D BB kicks, when distributed over several kicks, seems to be sufficient at least for the nominal LHC. This issue has to be reviewed for the LHC upgrade since the crossing angle will be larger. Lines depict all resonances up to $10^{\text {th }}$ order. 
The nominal BB tune shift is calculated as:

$$
\Delta Q=\frac{r_{p} N}{4 \pi \epsilon}
$$

A good approximation for alternating crossing Ref. [11] is:

$$
\Delta Q \approx \frac{r_{p} N \beta^{*}}{2 \pi \gamma \sigma^{*} \sqrt{\sigma^{* 2}+\theta^{2} \sigma_{z}^{2} / 4}}
$$

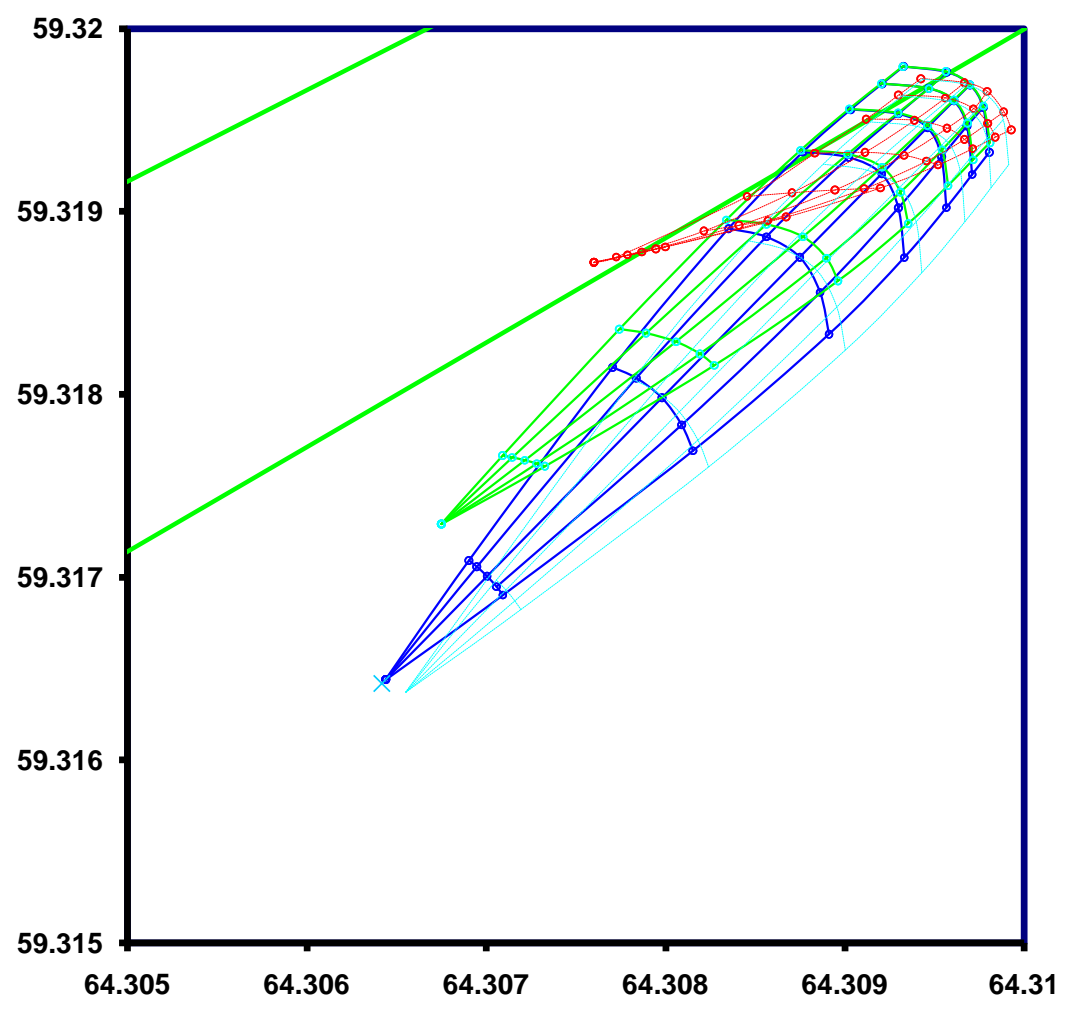

Figure 18: LHC head-on BB in IP1 only but with nominal crossing angle. Dark blue shows the 4D $\mathrm{BB}$ ( 1 slice only) with deltap $=0$, when large deltap $=0.00027$ is used the weak coupling between transverse and longitudinal phase space leads to the slightly shifted light blue tune footprint. For a single head-on BB there is no longer a compensation since the crossing angle in one plane only: in green is shown the effect of the $6 \mathrm{D}$ BB (13 slices) at deltap $=0$, while for deltap $=0.00027$ the shift and distortion is significant (shown in red). The bluish " $\mathrm{X}$ " symbol shows the nominal BB tune-shift for one BB interaction. Lines depict all resonances up to $10^{\text {th }}$ order. 


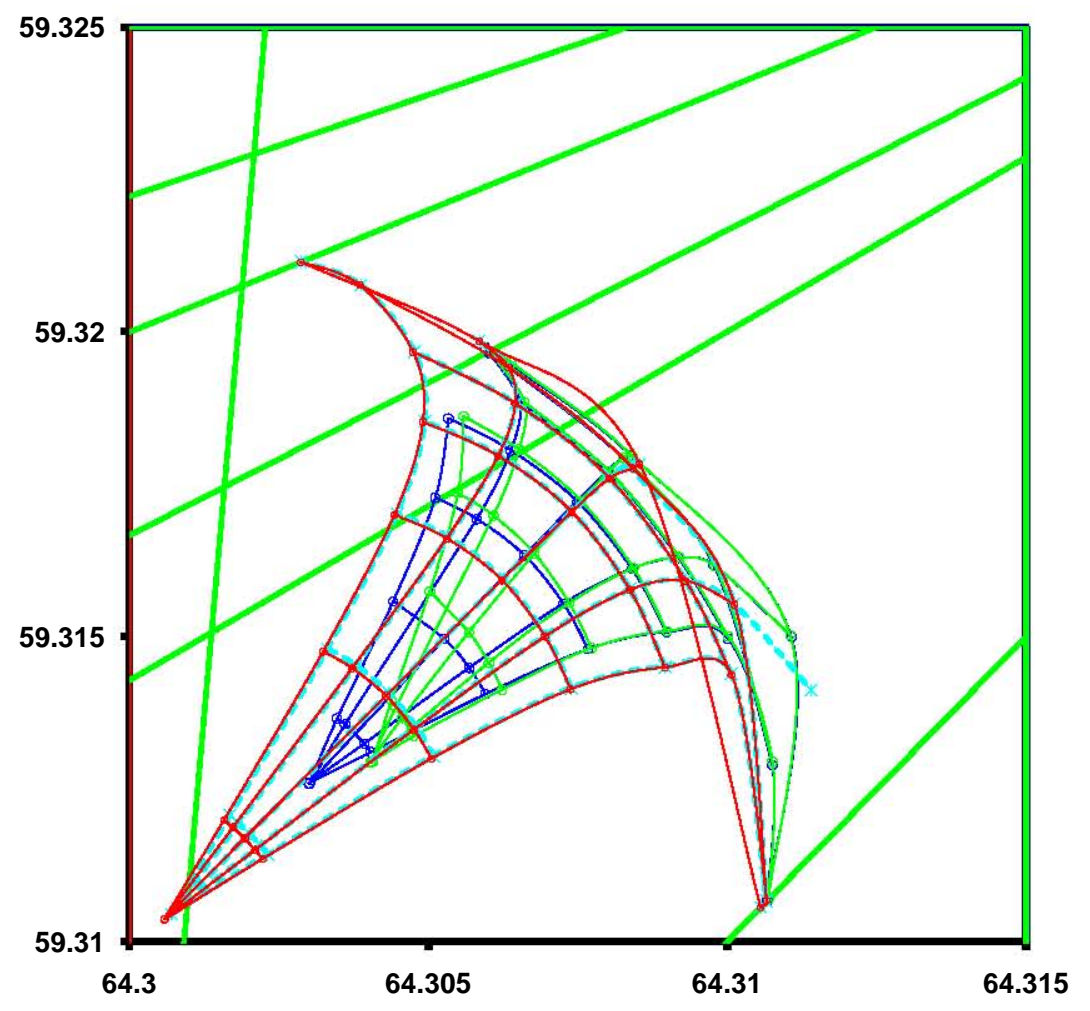

Figure 19: LHC head-on and parasitic BB kicks in all 4 IRs. The tune footprint for the 4D BB case ( 5 slices) and the $6 \mathrm{D}$ BB (13 slices) with deltap $=0$ are practically the same (red and dotted cyan footprint respectively). However for large deltap $=0.00027$ the tune footprint shrinks when in IP1 \& IP5 the 4D head-on BB kicks are replaced by $6 \mathrm{D}$ head-on BB kicks (blue footprint) and it shrinks further when all 4 IPs have $6 \mathrm{~d}$ head-on BB kicks (green footprint). In the $4 \mathrm{D}$ case a change of deltap just leads to a very small shift of the tune footprint as in the Fig. 18. All parasitic BB kicks are treated as 4D BB kicks. Lines depict all resonances up to $10^{\text {th }}$ order. 


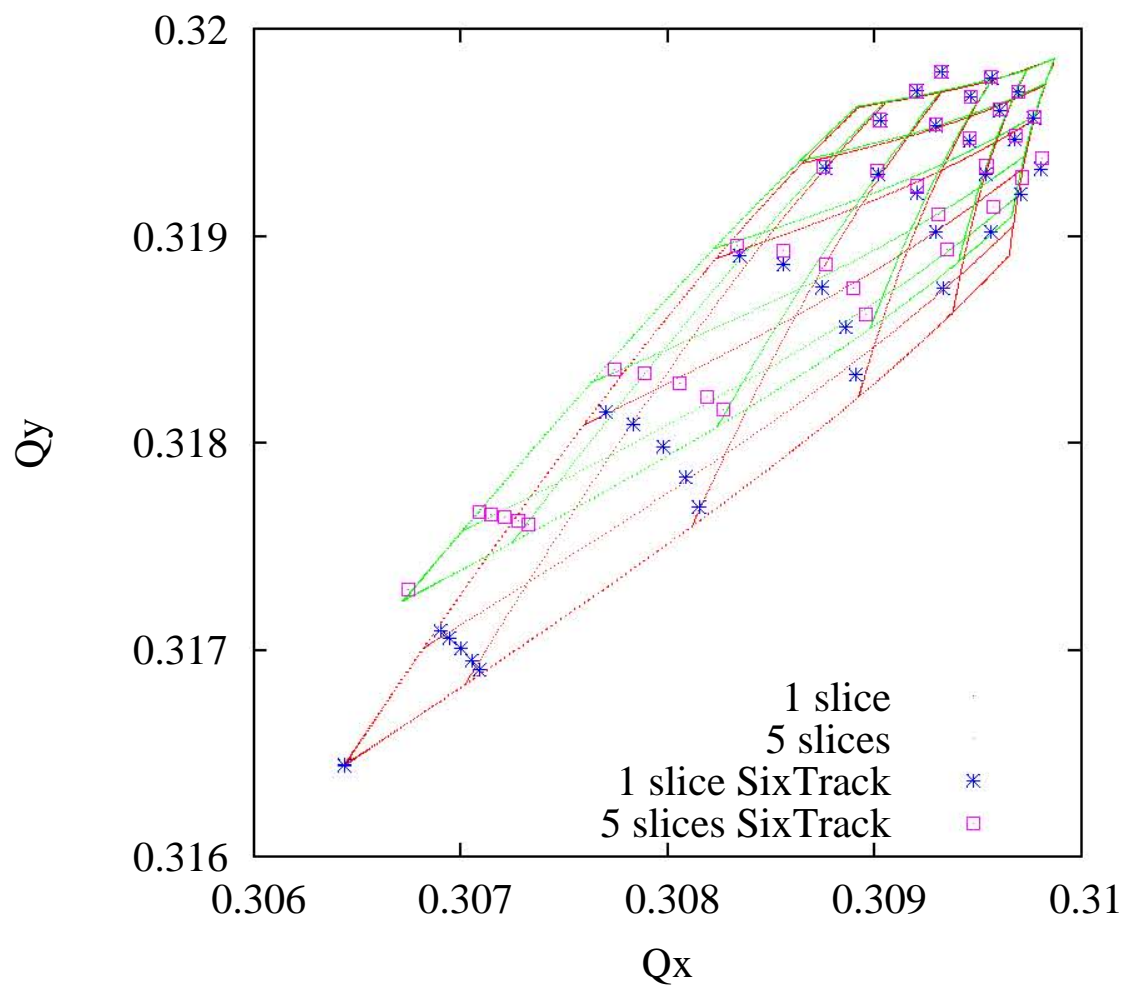

Figure 20: Comparison of SixTrack and Lifetrac in the case of LHC head-on with one IP. The tune footprints for the $4 \mathrm{D}$ BB case (1 slice, red lines Lifetrac) and the $6 \mathrm{~d}$ BB (5 slices, green lines Lifetrac) possess similar properties.

\section{LHC Dynamic Aperture for 4D \& 6D BB}

The results in this section are obtained with SixTrack only. Fig. 21 shows the comparison between the $4 \mathrm{D} \& 6 \mathrm{D}$ dynamic aperture (DA) over 10'000, 100'000 and 1'000'000 turns respectively. 


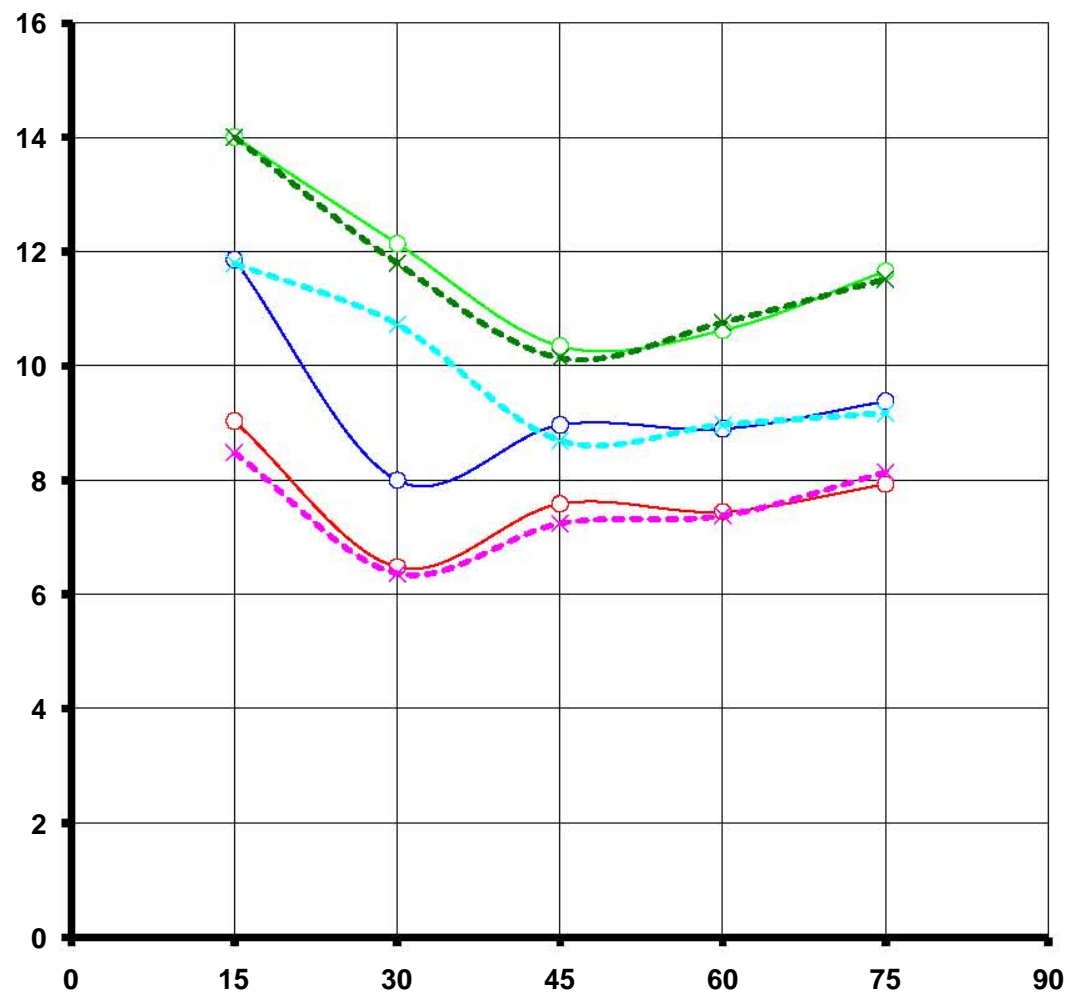

Figure 21: LHC dynamic aperture for the $4 \mathrm{D}$ and the $6 \mathrm{D}$ BB (13 slices) treatment. The curves in shades of green are DA for 10'000, bluish for 100'000 and reddish for 1'000'000 turns respectively. The solid lines with circles are from the $4 \mathrm{D}$ BB while the dashed curves with an " $\mathrm{X}$ " character are the results from $6 \mathrm{D}$ BB tracking.

It is interesting to note that the long-term DA is similar despite the fact that the tune footprints (see Fig. 19) are very different for the two types of BB treatments. This can only mean that the amplitude (in sigma) where the tune footprints fold over is more relevant for the particle stability than how far the tune footprint extends for the low amplitude particles.

\section{RHIC tune footprints}

Due to the absence of implementation of crossing-angle collisions in SimTrack, it was impractical to perform benchmarking with this code for the LHC case. We compared the tune footprints produced by SixTrack and SimTrack without and with beam-beam interactions with the Blue ring lattice for the RHIC $2011250 \mathrm{GeV}$ polarized proton run. We set the tunes in absence of BB to (28.695, 
29.685) and linear chromaticities to $(1,1)$. The nonlinear multipole field errors from interaction regions are included. The $\beta^{*}$ at interaction points IP6 and IP 8 are $0.7 \mathrm{~m}$. In the simulation study, we used one RF cavity whose voltage is set to $300 \mathrm{kV}$. The proton bunch intensity is $1.5 \times 10^{11}$. The bunch length is $0.45 \mathrm{~m}$. In the weak-strong beam-beam simulation, we split the strong beam into 13 slices in both codes. The caption describes the details of the plot.

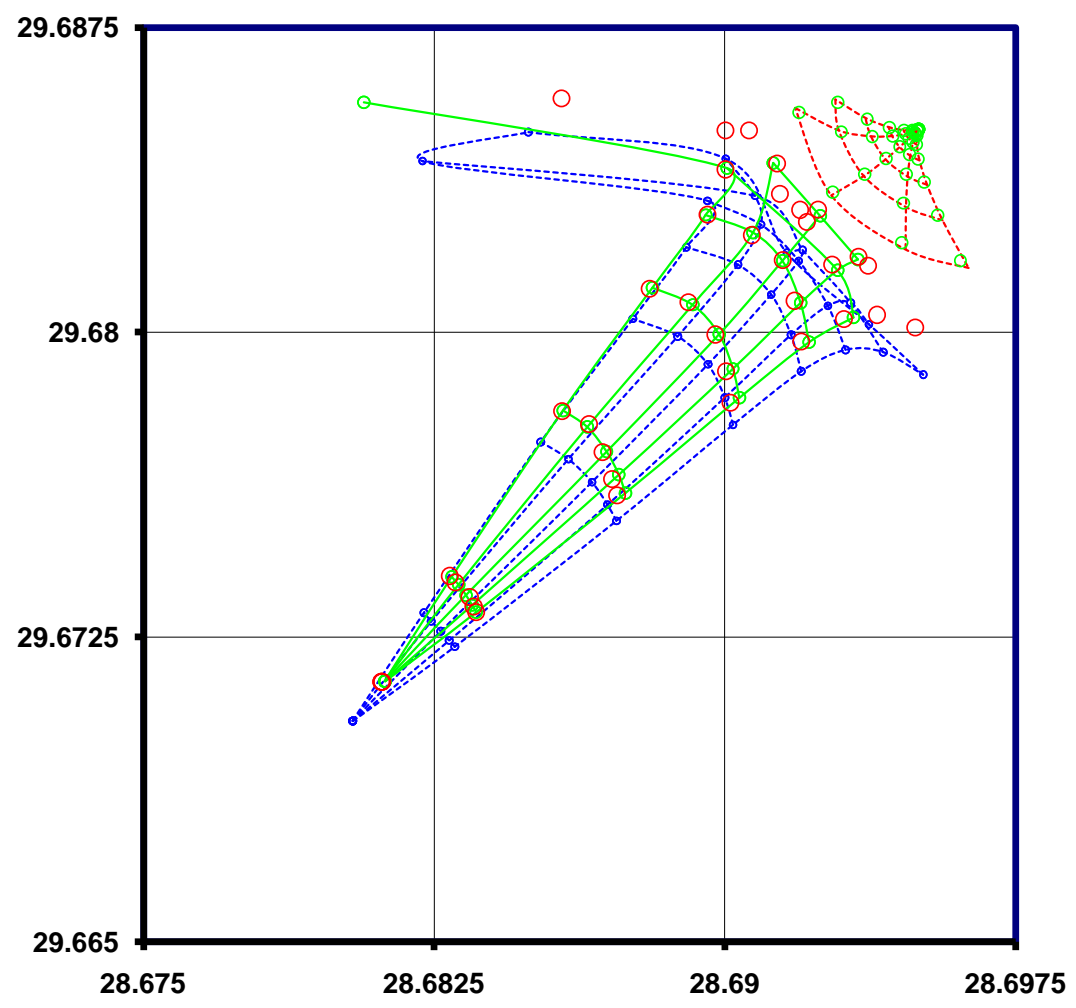

Figure 22: In broken red lines the SixTrack 4D case is shown without BB with data from SimTrack shown on top as green circles. The green solid lines show SixTrack calculations with 6D BB (13 slices). Also in this case the SimTrack results (red circles) are very close by. This tracking was done with deltap $=0.001$. As a comparison (broken blue line) the same case is shown for deltap $=0$. The deltap $=0.001$ tune footprint is shifted due to relatively large $\mathrm{Q}$ " but apparently the $6 \mathrm{D}$ BB treatment does not lead to an additional shrinking of the tune footprint. 


\section{Summary}

We performed single-particle tracking with three beam-beam codes, Lifetrac, SixTrack and SimTrack in a number of machine configurations for LHC and RHIC, and found the results to be in good agreement. In particular, the following cases were studied:

- 4D and 6D tracking in the LHC lattice with a full set of nonlinearities (SixTrack) and essential nonlinearities (Lifetrac), and beam-beam interaction switched off, produced the dynamic aperture values within $20 \%$ for a number of initial conditions.

- With beam-beam, the agreement between dynamical apertures given by SixTrack and Lifetrac is also good.

- LHC tune footprints produced by SixTrack and Lifetrac agree perfectly.

- RHIC tune footprints produced by SixTrack and SimTrack agree perfectly.

In addition to the code benchmarking, the important result obtained during these studies was the demonstration of similarity of dynamical aperture produced by $4 \mathrm{D}$ and $6 \mathrm{D}$ tracking for the LHC.

\section{References}

[1] D. Shatilov, et al., "Lifetrac Code for the Weak-Strong Simulation of the Beam-Beam Effects in Tevatron", PAC'05, Knoxville, TN, p. 4138.

[2] F. Schmidt, "SixTrack Version 4.2.13", CERN/SL/94-56 (AP), Update February 2011; Web page: http://www.cern.ch/frs.

[3] G. Ripken, "Nonlinear canonical equations of coupled synchro-betatron motion and their solution within the framework of a nonlinear 6-dimensional (symplectic) tracking program for ultra-relativistic protons", DESY 85-084 (1985); D.P. Barber, G. Ripken and F. Schmidt, "A nonlinear canonical formalism for the coupled synchro-betatron motion of protons with arbitrary energy", DESY 87-036 (1987); G. Ripken and F. Schmidt, "A symplectic six-dimensional thin-lens formalism for tracking", CERN/SL/95-12 (AP), DESY 95-063 (1995).

[4] M. Bassetti and G.A. Erskine, "Closed expression for the electrical field of a two-dimensional Gaussian charge", CERN-ISR-TH/80-06.

[5] K. Hirata, H. Moshammer, F. Ruggiero and M. Bassetti, "Synchro-Beam interaction", CERN SL-AP/90-02 (1990) and Proc. Workshop on Beam Dynamics Issues of High-Luminosity Asymmetric Collider Rings, Berkeley, 1990, ed. A.M. Sessler (AIP Conf. Proc. 214, New York, 1990), pp. 389-404; K. Hirata, H. Moshammer and F. Ruggiero, "A symplectic beam-beam interaction with energy change", KEK preprint 92-117 A (1992) and Part. Accel. 40, 205-228 (1993); K. Hirata, "BBC User's Guide; A Computer Code for Beam-Beam Interaction with a Crossing Angle, version 3.4", SL-Note 97-57 AP.

[6] L.H.A. Leunissen, F. Schmidt and G. Ripken, "6D Beam-Beam Kick including Coupled Motion", LHC Project Report 369, PRST-AB, Volume 3, Number 12. 
[7] Eric Mcintosh, "Massive Tracking on Heterogeneous Platforms", 2006 ICAP Conference in Chamonix, France.

[8] R.D. Ruth, "A canonical Integration Technique", IEEE Trans. Nucl. Sci., vol. NS-30, PP.26692671 (1983).

[9] J. Bengtsson, "The Sextupole Scheme for Swiss Light Source (SLS): An Analytic Approach", SLS Note 9/97, March 1997.

[10] Y-J. Kim and T. Sen, PRST-AB 12, 031001 (2009).

[11] F. Ruggiero and F. Zimmermann, "Luminosity optimization near the beam-beam limit by increasing bunch length or crossing angle", PRSTAB, Volume 5, 061001, 2002. 